Thus any (standard) regenerative event can be represented by a Markov process on a continuous state space. It follows, for example, that the fact that $p_{00}(t)$ is almost everywhere differentiable is a consequence of the regenerative property of the state 0 , but the deeper result that $p_{00}(t)$ is everywhere differentiable requires also the discrete nature of the state space.

It is possible to extend the whole theory to take in properties of several states simultaneously, by considering systems of regenerative events. In particular, we can examine the transition probabilities $p_{i j}(t)(i \neq j)$ of a Markov chain. The theory may also be applied to certain Markov processes with continuous state space, and so, via the method of supplementary variables, to some non-Markovian processes.

It is hoped to publish elsewhere a detailed account of the theory summarised here, and of its various applications.

I am deeply grateful to Professor D. G. Kendall for much helpful discussion, and also to the Department of Scientific and Industrial Research for financial support.

\title{
REFERENCES
}

1. K. L. Chung, Markov chains with stationary transition probabilities, Springer, Berlin, 1960.

2. W. Feller, An introduction to probability theory and its applications, Wiley, New York, 1957.

University of Cambridge, Cambridge, England

\section{ORIENTABLE EMBEDDING OF CAYLEY GRAPHS}

BY WILLIAM GUSTIN

Communicated by J. W. T. Youngs, November 14, 1962

I present a method whereby a polygonal embedding of a Cayley graph in a closed oriented polyhedral surface may be represented as the dual of a quotient embedding of a quotient graph and diagrammed as a linked network of circuits carrying currents satisfying Kirchhoff's node law. By this means, devised to aid construction of triangular embeddings of a complete $n$ node to affirm Heawood's map color conjecture [3] in Heffter's dual formulation [4] for those cases $n \equiv 0,3,4,7 \bmod 12$ where such triangulation is compatible with Euler's polyhedral formula, I have been able to solve the cases $n \equiv 3,4,7 \bmod 12$, unaware that Ringel [5] had already resolved cases $n \equiv 3,7$ by a similar though less developed method. Case $n \equiv 0$ remains 
undecided. I here outline the method of embedding by quotient network and illustrate it by one example from each of the solved cases. Full exposition will be published elsewhere.

An arc graph $\mathfrak{g}$ is to consist of a finite set of elements named arcs and a finite set of elements named nodes, together with a mapping called stem from arcs onto nodes and an involutory permutation of arcs called reverse, the reverse of an arc $a$ being denoted by ${ }^{*} a$. An arc is to have as tip the stem of its reverse; a node is to have as hub the set of arcs which stem from it and as border the set of tips of these hub arcs.

Recently Edmonds [2] has shown that any polygonal embedding of a connected graph in a closed oriented polyhedral surface is combinatorially characterized by assigning at each node a cyclic order on the arcs stemming therefrom. Hence for an arc graph $\mathfrak{g}$ such an embedding may be regarded as a permutation of arcs, here also called rotation, which has the hub sets of $\mathfrak{g}$ as cycle sets, that is, as minimal nonnull fixed sets.

To any rotation $p$ on $g$ associate another permutation $p^{*}$ of arcs, called circulation, defined by $p^{*}(a)=p\left({ }^{*} a\right)$, and call the cycle sets of $p^{*}$ the circuits or faces of $p$. The dual graph $\mathfrak{g}^{*}$ of $\mathfrak{g}$ by $p$ has the same arcs and reverse as $g$ but its node-hubs are the circuits of $p$. Thus $p^{*}$ is a rotation on $\mathfrak{g}^{*}$ and $p^{* *}=p$ the associate circulation.

Let $\gamma$ be a finite group on $n$ elements $x$ and $\Delta$ a subset of $m$ elements $\delta$ called currents which generates $\gamma$ and is symmetric with respect to inverse. The Cayley graph (cf. [1]) g of $\gamma$ by $\Delta$ has as nodes the $n$ elements $x$ of $\gamma$, and the $n m$ pairs $(x, \delta)$ as arcs. The arc $a=(x, \delta)$ has $x$ as stem and the arc ${ }^{*} a=\left(x \delta, \delta^{-1}\right)$ as reverse, so has tip $x \delta$; we shall moreover say that it carries the current $\partial a=\delta$ and has the arc $\omega a=(\omega x, \delta)$ as $\omega$ translate.

A rotation $p$ on $\mathfrak{g}$, being cyclic on the hub $(x, \Delta)$ of arcs stemming from each node $x$, may equally well be represented by the cyclic permutation $\pi_{x}$ of currents $\Delta$ defined by $\pi_{x}(\delta)=\partial p(x, \delta)$ or by the cyclic permutation $p_{x}=x \pi_{x} x^{-1}$ of the border $x \Delta$ of $x$. Thus $p:(x, \delta)$ $\rightarrow\left(x, \delta^{\prime}\right)$ may be rendered as $\pi_{x}: \delta \rightarrow \delta^{\prime}$ or as $p_{x}: x \delta \rightarrow x \delta^{\prime}$.

A translation $\omega$ in the center of $\gamma$ will be called invariant for $p$ if $p \omega=\omega p$, that is, if $\pi_{\omega x}=\pi_{x}$ or $p_{\omega x} \omega=\omega p_{x}$ for all $x$. Let $\Omega$ be the group of all such $\omega, \Gamma$ the quotient group of $\gamma \bmod \Omega$, and $N$ the index of $\Omega$ in $\gamma$. The quotient graph $\&$ of $g \bmod \Omega$ is the Schreier graph (cf. [1]) of $\Gamma$ by $\Delta$ having as nodes the $N$ cosets $X$ of $\Gamma$ and the $N m$ pairs $(X, \delta)$ as arcs.

The rotation $p$ on $\mathfrak{g}$ induces a rotation $P$ on (f) such that $\Pi_{X}=\pi_{x}$ for all $x$ in $X$. Moreover, $p$ on $g$ can be recovered from $P$ on (B) or, often more conveniently, from $P^{*}$ on $\mathbb{S}^{*}$ as described below for the 
regular unfurled case when all cycles of $p^{*}$ and $P^{*}$ have the same length $l$.

The Cayley graph $\mathrm{g}$ of a group $\gamma$ by a set $\Delta$ of $m$ currents can be regularly embedded in a closed oriented polyhedral surface so that all faces are l-gons, if there exists a central subgroup $\Omega$ of index $N$ in $\gamma$ and an arc graph (5* with rotation $P^{*}$ and current flow $\partial$ on it such that:

(1) The graph \&5* has $\mathrm{Nm}$ arcs and $\mathrm{Nm} / \mathrm{l}$ nodes, with l arcs stemming from each node.

(2) The rotation $P^{*}$ on $5^{*}$, cyclic on the larcs from each node, induces a circulation $P^{* *}=P$ on $\$ 5^{*}$ having $N$ circuits each composed of $m$ arcs.

(3) The currents on each circuit assume all $m$ values of $\Delta$.

(4) An arc and its reverse carry inverse currents.

(5) The currents taken in cyclic order of rotation round each node have unit product.

(6) The $N$ circuits can so be labeled by the $N$ cosets mod $\Omega$ that an arc on circuit labeled $X$ with reverse on circuit labeled $Y$ has current in the coset $X^{-1} Y$.

The current cycle $\Pi_{X}$ on the circuit labeled $X$ of $\$ 3 *$ then determines the current rotation $\pi_{x}$ round the node $x$ of $\mathfrak{g}$ by putting $\pi_{x}=\Pi_{x}$ for all $x$ in the coset $X$.

The complete $n$ node may be realized as the Cayley graph on the additive group of integers $\bmod n$ with all $m=n-1$ nonzero elements as currents. The accompanying Figures 1, 2, 3 illustrate oriented triangular embedding of such graphs in a surface of genus $(n-3)(n-4) / 12$ for the values $n=43,28,27$ exemplifying cases $n \equiv 7,4,3$ mod 12 of index $N=1,2,3$ respectively. Each figure is composed of two diagrams: one showing currents, the other showing rotation and circulation. Since currents on reverse arcs must balance, the current diagram need only exhibit one of a pair of reverse arcs; note that for even $n$ the arc carrying the self-inverse current $n / 2$ must be self-reverse. The rotation which cyclicly permutes the $l=3$
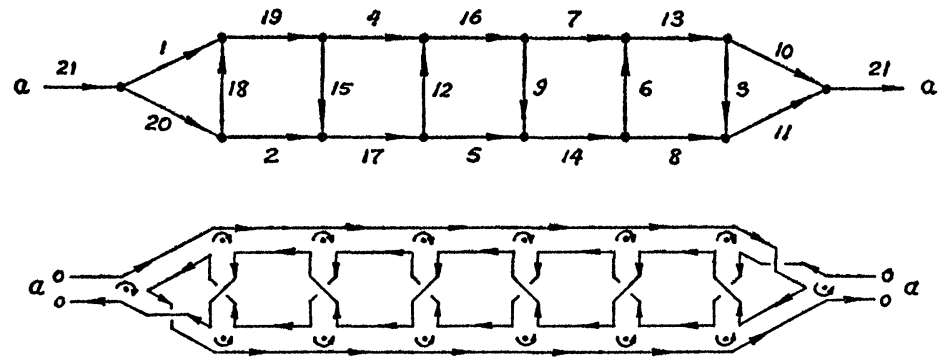

FIGURE $1(n=43, N=1)$ 

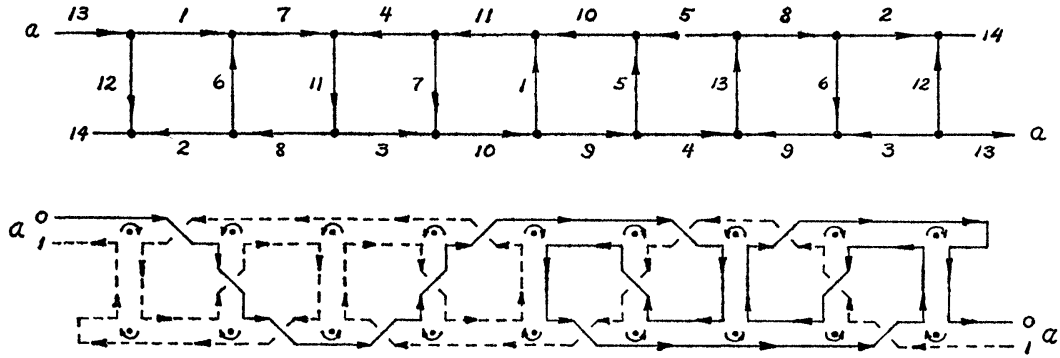

FIGURE $2(n=28, N=2)$
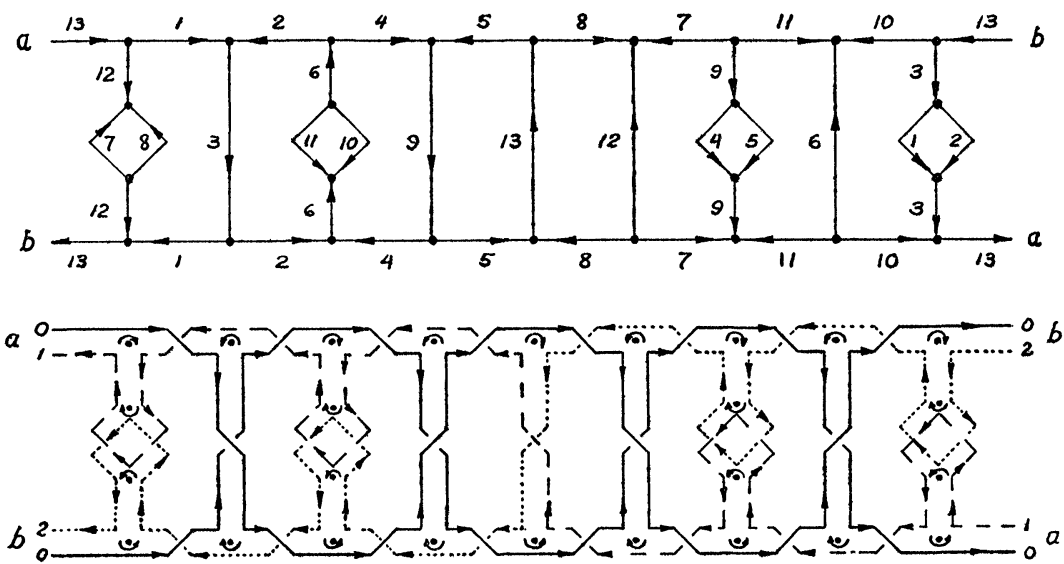

FIGURE $3(n=27, N=3)$

arcs stemming from each node is represented by a curved arrow round that node; the associate circuits are labeled by coset representatives 0,1 , or 2 . A few arcs have been broken as indicated to display the diagrams as linear bands.

\section{REFERENCES}

1. H. S. M. Coxeter and W. O. J. Moser, Generators and relations for discrete groups, Ergebnisse Math., Neue Folge 14, Springer, Berlin, 1957.

2. J. R. Edmonds, A combinatorial representation for polyhedral surfaces, Notices Amer. Math. Soc. 7 (1960), 646.

3. P. J. Heawood, Map-colour theorem, Quart. J. Math. 24 (1890), 332-338. 508.

4. L. Heffter, Über das Problem der Nachbargebiete, Math. Ann. 38 (1891), 477-

5. G. Ringel, Über das Problem der Nachbargebiete auf orientierbaren Flächen, Abh. Math. Sem. Univ. Hamburg 25 (1961), 105-127.

INDIANA UNIVERSITY AND

National Bureau of Standards 